\title{
Application of Fuzzy C-means clustering algorithm in image segmentation
}

\author{
Rongchuan Guo \\ Jiangxi University Of Traditional Chinese Medicine Yao Hesheng Research \\ Center,JiangxiNanchang 330006, China \\ Email: rongchuan613@qq.com
}

Keywords: Interval type-2 fuzzy sets; means clustering algorithm; Image Segmentation.

\begin{abstract}
Data mining was a core process step in the whole data processing system. The aim is to use specific data mining algorithms to extract knowledge of interest. The user from the database and represented in a certain way, such as the generation rules. Typically, data mining algorithms can be implemented features include: class concept descriptions, association rules, classification regression, cluster analysis, sequence timing analysis, and isolated point analysis. This paper describes one of the most common algorithms based on fuzzy clustering algorithm, which called FCM objective function. FCM algorithm discussed two types of improvement ideas: First, the introduction of clustering validity function in FCM algorithm process to determine the number of clusters c. Second is the evolutionary computation is introduced into the FCM algorithm. On this basis, an improved FCM algorithm, namely through the introduction of simulated annealing particle swarm algorithm FCM is improved to reduce the impact of isolated points on the cluster center.
\end{abstract}

\section{Introduction}

Fuzzy sets a value which is determined as the degree of membership fuzzy sets to describe the uncertainty of traditional fuzzy system based on a fuzzy set is constructed, using the corresponding fuzzy logic reasoning and precise to achieve a specific system function, but a type Fuzzy sets in dealing with the uncertainty of the actual object limitations. Enhanced systems approach can improve the ability of the ambiguity of the system dealing with uncertainty, Zadeh [1] ambiguity extent of the problem in the concept of fuzzy sets proposed had mentioned the collection of a fuzzy set extension, given the set membership the degree of blurring value, so that the collection descriptions are more ambiguity, this extension of fuzzy set is called type-2 fuzzy sets. Mendel J M presented two complete systems approach based on fuzzy type-2 fuzzy sets on that type-2 fuzzy systems have broader application prospects [2].

By Fuzzy Clustering is the concept of fuzzy sets applied to traditional clustering analysis, so that the data set in the packet to determine the membership by the membership function, that is, objects in the membership of each packet is a continuous interval between a value [0,1], a plurality of clusters belonging to different degrees, but not deterministic clustering two-valued logic 0 or 1 . Fuzzy Clustering advantage lies in the ability to adapt very good separation of the data and class, which allows the ambiguity of the nature of the data, to describe the data structure provides detailed information [3, 4]. Because the fuzzy clustering degree of uncertainty obtained samples belonging to each category, expressed sample generic vagueness, namely to establish a sample in relation to the description of categories of uncertainty, more objectively reflect the real world, thus becoming clustering one of the main research [5-7].

Fuzzy clustering segmentation method must be given initial cluster centers. According to the mathematical theory, any convergence of an iteration and the last sequence, if the initial value is closer to the final iteration, then convergence result, the speed of convergence will significantly increase the number of iterations will be more substantial decrease. In addition, if the initial value of clustering iterative close to a local extreme value, then it is likely to end up in local minima, which are not global optimum. Therefore FCM algorithm is very sensitive to initial values. In this paper, fuzzy $\mathrm{C}$ - means clustering algorithm to image segmentation. Through the improved algorithm for clustering 
pixels in the image, the improved algorithm can find the images more scientific and accurate segmentation.

\section{Basic theory and methods}

\subsection{Standard FCM algorithm}

FCM algorithm is based on the objective function of the fuzzy clustering method, that is, the clustering normalized knot non-linear regulation to bring about bundles plan issues through optimization solver obtain data sets fuzzy classification and clustering. FCM algorithm by iterative optimization objective function, the fuzzy membership matrix U represents the classification results. For image segmentation, the data set is the pixel values of the image, pixel by FCM algorithm will tap into the gray value c class to give c clustering centers and the corresponding fuzzy membership matrix. FCM algorithm objective function is defined as:

$$
\begin{aligned}
& \left\{\begin{array}{l}
J_{m}(U, V)=\sum_{k=1}^{n} \sum_{i=1}^{c}\left(\mu_{i k}\right)^{m}\left(d_{i k}\right)^{2}, m \in[1, \infty) \\
\text { s.t. } U \in M_{f c}
\end{array}\right. \\
& M_{f c}=\left\{U \in R^{c n} \mid \mu_{i k} \in[0,1], \forall_{i, k} ; \sum_{i=1}^{c} \mu_{i k}=1, \forall_{k} ; 0<\sum_{k=1}^{n} \mu_{i k}<n, \forall_{i}\right\}
\end{aligned}
$$

Where: $V$ represents the center of the cluster sample; $d_{i k}=\left\|x_{k}-v_{i}\right\|^{2}$ indicates the distance the i-th cluster center to the k-th sample; m called fuzzy weighted index, also known as the smoothing parameter that indicates the degree of fuzzy fuzzy clustering algorithm. FCM specific steps are as follows:

1) Given the number of categories $\mathrm{c}$ and clustering parameter $\mathrm{m}, 2<_{\mathrm{c}}<\mathrm{n}, \mathrm{n}$ is the total number of data sets iteration stops threshold iteration counter $\mathrm{l}=0$;

2) Initialization fuzzy partition matrix $\mathrm{U}, U^{(l)}=\left[\mu_{i k}(i, j)^{(l)}\right]$

3) Represented by the formula (3) or update cluster centers calculate $\mathrm{V}^{(1)}$;

$$
\begin{aligned}
& v_{i}^{l}=\sum_{k=1}^{n}\left(\mu_{i k}^{(l)}\right)^{m} x_{k} / \sum_{k=1}^{n}\left(\mu_{i k}^{(l)}\right)^{m} \\
& \mu_{i k}^{(l+1)}=1 / \sum_{j}^{c}\left(d_{i k}^{(l)} / d_{j k}^{(l)}\right)^{2 /(m-1)}
\end{aligned}
$$

4) Meet $\left\|V^{(l+1)}-V^{(l)}\right\|<\varepsilon \rightarrow \infty$ iteration stops, otherwise $\mathrm{l}=\mathrm{l}+1$ transfer section (3) steps.

\subsection{Proposed of type-2 fuzzy theory}

As shown in Figure 1, so as to strengthen the ability of fuzzy set description. Clustering algorithm in image segmentation is divided into two areas, one of the algorithm itself conduct research to improve the image segmentation results. The above methods are of this kind. Second, the study in terms of image feature extraction. Background research in this area comes from the degraded image segmentation, these are some of the degraded image blurred images in uncontrollable situation was such that image noise, objectives and background of overlapping images, boundary is not clear images. 


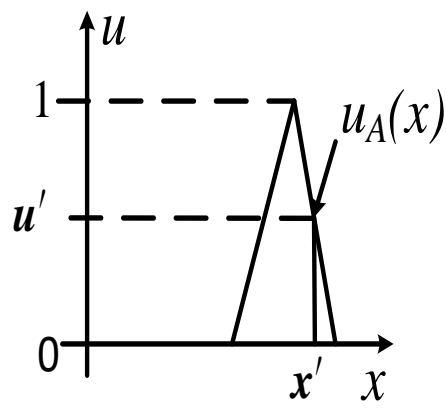

(a) A set of fuzzy membership functions

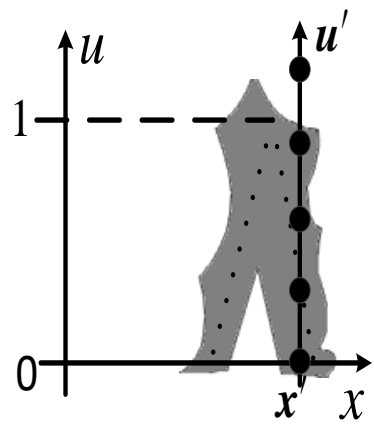

(b) Fuzzy membership function

Figure 1. Fuzzy set extended membership function

\section{Experiment and results}

\subsection{Results of fuzzy training data}

It is the algorithm and fuzzy C- means clustering algorithm compares experiment given the same initial membership matrix using different algorithms for image segmentation experiments. Results of fuzzy training data were shown in Table 1. Experimental parameters are as follows: Category Number c take 10 population size $\mathrm{N}$ is 20 , the inertia weight from $\mathrm{w}=0.9$ decreases linearly to $\mathrm{w}=$ 0.5 , learning factor $1 \mathrm{c}$ and $2 \mathrm{c}$ have taken 2 , maximum number of iterations 1000 , fuzzy index $\mathrm{m}=2$ The maximum length $L=20$. The results were shown in Figure 2.

Table 1. Results of fuzzy training data

\begin{tabular}{c|c|c|c|c}
\hline \multirow{2}{*}{ Time } & $U_{1}$ & $\ldots$ & $U_{i}$ & \multirow{2}{*}{ Fuzzy Sets } \\
\cline { 2 - 4 } & $\boldsymbol{u}_{1,1}^{t}, \ldots, \boldsymbol{u}_{1, n_{1}}^{t}$ & $\ldots$ & $\boldsymbol{u}_{i, 1}^{t}, \ldots, \boldsymbol{u}_{i, n_{1}}^{t}$ & \\
\hline $\mathrm{S}(1)$ & $\boldsymbol{u}_{1,1}^{1}, \ldots, \boldsymbol{u}_{1, n_{1}}^{1}$ & $\ldots$ & $\boldsymbol{u}_{i, 1}^{1}, \ldots, \boldsymbol{u}_{i, n_{1}}^{1}$ & $A_{\bar{m}}(1)$ \\
$\mathrm{S}(2)$ & $\boldsymbol{u}_{1,1}^{2}, \ldots, \boldsymbol{u}_{1, n_{1}}^{2}$ & $\ldots$ & $\boldsymbol{u}_{i, 1}^{2}, \ldots, \boldsymbol{u}_{i, n_{1}}^{2}$ & $A_{\bar{m}}(2)$ \\
$\vdots$ & $\vdots$ & $\vdots$ & $\vdots$ & $\vdots$ \\
$\mathrm{S}(\mathrm{N}-1)$ & $\boldsymbol{u}_{1,1}^{N-1}, \ldots, \boldsymbol{u}_{1, n_{1}}^{N-1}$ & $\ldots$ & $\boldsymbol{u}_{i, 1}^{N-1}, \ldots, \boldsymbol{u}_{i, n_{1}}^{N-1}$ & $A_{\bar{m}}(N-1)$ \\
$\mathrm{S}(\mathrm{N})$ & $\boldsymbol{u}_{1,1}^{N}, \ldots, \boldsymbol{u}_{1, n_{1}}^{N}$ & $\ldots$ & $\boldsymbol{u}_{i, 1}^{N}, \ldots, \boldsymbol{u}_{i, n_{1}}^{N}$ & $A_{\bar{m}}(N)$ \\
\hline
\end{tabular}

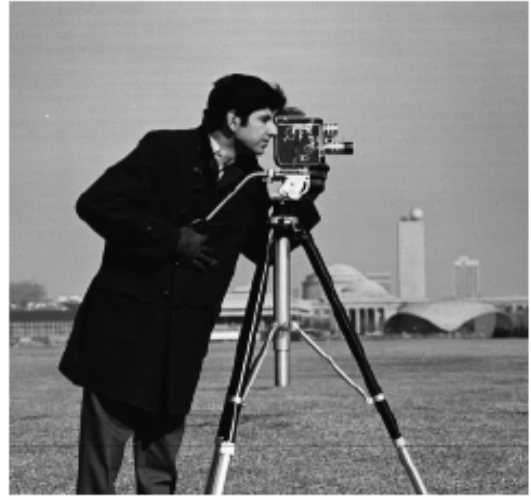

(a)Standard cameraman image

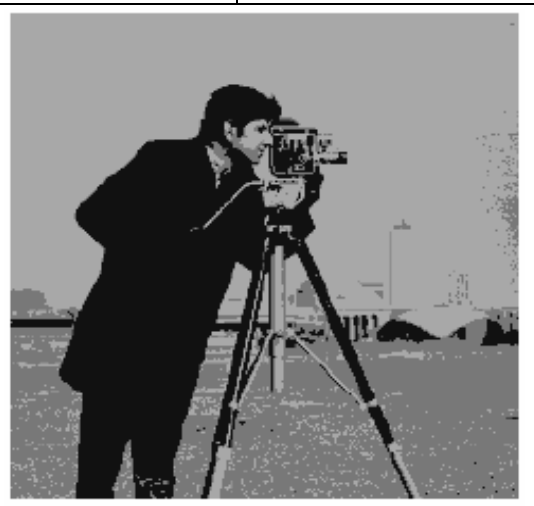

(b) Fuzzy C- Means clustering segmentation

Figure 2. Image segmentation results 


\subsection{Discriminant validity of FCM algorithm}

Although the FCM algorithm is an unsupervised machine learning algorithm since, however, there are two arguments in front of the cluster analysis must be given an appropriate assignment, namely fuzzy clustering weighted index $\mathrm{m}$ and the number of categories c, otherwise it will affect the FCM algorithm analysis of the effect of a direct impact on reasonable explanation cluster analysis results. In order to be able to carry out $m$ and c preferably, it must be some function of determining the validity of clustering to be selected. Clustering validity judgment algorithm flow was shown in Figure 3.

Figure 3. Clustering validity judgment algorithm flow

\section{Summary}

In this paper, FCM algorithm for image segmentation, in order to resolve uncertainties traditional FCM algorithm can not handle, compared to the FCM algorithm, parameter $\mathrm{m}$ using precise value, FCM leads $m$ uncertain domain, to better address the value of $m$ uncertainty segmentation proved FCM noise and other uncertainties treatment is better than FCM algorithm. Fuzzy clustering algorithm for image segmentation when considering only the image pixel gray-scale information, ignoring the correlation between structure of image pixels. To this end a number of studies on the FCM algorithm to make improvements, add image spatial information, improved FCM algorithm proved to have better segmentation.

\section{Acknowledgement}

This research was supported by the foundation of Jiangxi University Of Traditional Chinese Medicine(No.2013YHS012) . 


\section{References}

[1] Gong M, Liang Y, Shi J, et al. Fuzzy c-means clustering with local information and kernel metric for image segmentation[J]. IEEE Transactions on Image Processing, 2013, 22(2): 573-584.

[2] Feng Y, Guo H, Zhang H, et al. A modified fuzzy c-means method for segmenting MR images using non-local information[J]. Technology and Health Care, 2016 (Preprint): 1-9.

[3] Kaur P, Soni A K, Gosain A. RETRACTED: A robust kernelized intuitionistic fuzzy c-means clustering algorithm in segmentation of noisy medical images[J]. Pattern Recognition Letters, 2013, 34(2): 163-175.

[4] Zheng Y, Jeon B, Xu D, et al. Image segmentation by generalized hierarchical fuzzy C-means algorithm[J]. Journal of Intelligent \& Fuzzy Systems, 2015, 28(2): 961-973.

[5] Huang C W, Lin K P, Wu M C, et al. Intuitionistic fuzzy c-means clustering algorithm with neighborhood attraction in segmenting medical image[J]. Soft Computing, 2015, 19(2): 459-470.

[6] Benaichouche A N, Oulhadj H, Siarry P. Improved spatial fuzzy c-means clustering for image segmentation using PSO initialization, Mahalanobis distance and post-segmentation correction[J]. Digital Signal Processing, 2013, 23(5): 1390-1400.

[7] Zhao F, Jiao L, Liu H. Kernel generalized fuzzy c-means clustering with spatial information for image segmentation[J]. Digital Signal Processing, 2013, 23(1): 184-199. 